\title{
1 Host-pathogen co-existence incurs reproductive costs
}

2

3 Diederik Strubbe $^{\mathrm{a}^{*}}$, Roel Haesendonck ${ }^{\mathrm{b}^{*}}$, Elin Verbrugghe ${ }^{\mathrm{b}}$, Luc Lens $^{\mathrm{a}}$, Richard Ducatelle ${ }^{\mathrm{b}}$,

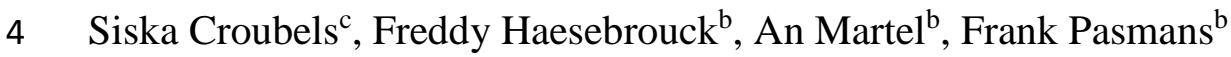

5

$6 \quad{ }^{a}$ Department of Biology, Terrestrial Ecology Unit, Faculty of Sciences, Ghent University, K.L.

7 Ledeganckstraat 35, 9000 Ghent, Belgium.

8

9 bepartment of Pathology, Bacteriology and Avian Diseases, Faculty of Veterinary Medicine,

Ghent University, Salisburylaan 133, 9820 Merelbeke, Belgium.

'Department of Pharmacology, Toxicology and Biochemistry, Faculty of Veterinary Medicine,

Ghent University, Salisburylaan 133, 9820 Merelbeke, Belgium.

* equal contribution

Corresponding author: diederik.strubbe@ugent.be, frank.pasmans@ugent.be 


\section{Abstract}

Widespread endemism of host-adapted pathogens poses a heavy burden on animal and human health. Mechanisms underpinning long-term host pathogen co-existence and concurrent costs are poorly understood. We use infections in pigeons with pathogenic, pigeon adapted Salmonella Typhimurium to explain how host and pathogen trade-offs and benefits sustain long-term pathogen endemism. An experimentally infected group of pigeons that was studied for 15 months showed that pathogen persistence decreased host condition and reproductive success, but conferred protection against Salmonella-induced clinical disease. The relevance of these findings was confirmed in nature, where this pathogen was shown to widely occur in feral pigeons (Columba livia), yet without clinical disease. Pathogen transmission and longterm persistence were associated with intermittent faecal shedding, which markedly increased during crop feeding and natural stress periods. Exploiting host specific traits in the presence of protective host population immunity thus facilitates long-term co-existence, be it at a significant reproductive cost.

(1)

\section{Abbreviations}

50 CFU: Colony Forming Units; IgY: Immunoglobulin Y; OD: Optical Density; PT: Phage Type 


\section{Introduction}

While limiting the impact of exposure to pathogens presents a continuous challenge to life on earth, pathogens face a similar struggle to assure their survival and persistence. Globally, bacterial pathogens that depend on a narrow host range as primary niche pose a heavy burden on animal and human health. Pronounced host adaptation requires maximizing pathogen persistence and transmission opportunities while limiting the negative impact on host populations [1,2]. Although key in epidemiology, the mechanisms sustaining endemism of host-adapted bacterial pathogens in the host population and associated trade-offs are currently poorly understood $[3,6,8,9]$ hampering attempts for endemic disease mitigation $[6,10]$. The underpinning infection dynamics are supposedly driven by host factors (e.g. age, stress and immunity), environment (e.g. biotic and abiotic factors such as food availability, stress, population size and density and pathogen reservoirs) and pathogen determinants such as level of host adaptation, virulence factors and transmission mechanism [3,4]. Adverse effects of endemic infections on the host may vary at the individual and population level [5-7] but overall could be expected to be tempered, avoiding long-term host population declines with substantial reduction of the pathogen's niche.

We here use Salmonella as a model organism to study mechanisms of pathogen endemism. Salmonella enterica subspecies enterica consists of over 2500 serovars, several of which are known to be endemic in various animal populations and even humans $[1,8,11-13]$. The host-adapted Salmonella enterica subspecies enterica serovar Typhimurium (Salmonella Typhimurium) PT99 strain in pigeons (Columba livia) is characterized by a very narrow host spectrum and pigeons are considered its main host $[1,14,15]$. Similar to other host-adapted Salmonella serovars, the course of an infection with this lineage may vary from subclinical to 
a typhoid fever-like disease, with a tropism for the host gonads, suggesting that vertical transmission occurs $[14,15]$.

In a one year infection trial, we identified factors driving Salmonella infection dynamics during endemism and associated costs and benefits for the host population. We determined to what extent Salmonella exploits host specific traits associated with stress (molting, breeding) or reproduction (crop feeding of nestlings, vertical transmission through infected eggs) that may increase bacterial shedding and promote transmission. Finally, we conducted a field study across Flanders (northern Belgium) in which we estimated Salmonella prevalence and clinical impact in populations of feral pigeons to confirm the validity of the experimental results.

\section{Results}

\section{Subclinical Salmonella infections are endemic in feral pigeon populations}

Salmonella Typhimurium occurrence was found in all four studied urban pigeon populations. High risk of exposure to Salmonella was demonstrated by marked seroprevalence (33.83\%, range: $13.33 \%-56.41 \%$, see Fig. 1; average true prevalence varies between $5.0 \%$ and $78.4 \%, 95 \%$ confidence interval, see table S1a). 


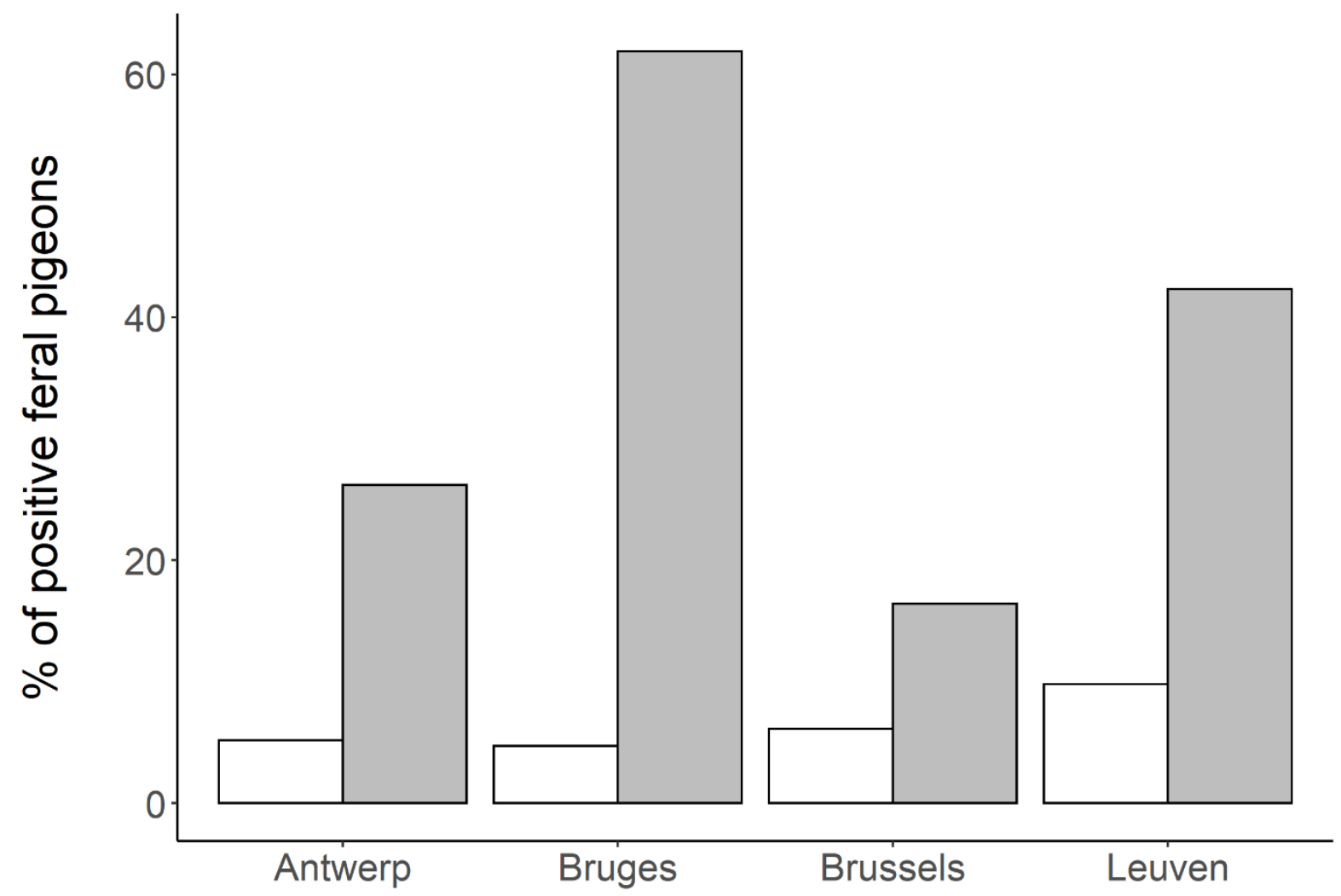

City

Fig 1. Faecal Salmonella shedding and presence of serum anti-Salmonella antibodies in feral pigeons. White bars represent the percentage of pigeons from which Salmonella Typhimurium was isolated (on average 3.76\%), and grey bars the percentage of Salmonella seropositive pigeons (on average $33.83 \%$ ) in a given population.

In all populations, a low percentage of pigeons shed low numbers of Salmonella Typhimurium in the faeces (average apparent prevalence: $3.76 \%$, range: $2.56 \%-6.90 \%$; average true prevalence varies between $0.8 \%$ and $8.4 \%, 95 \%$ confidence interval, table S1b). There was no significant correlation between health status, body constitution score and the presence of faecal Salmonella or serum anti-Salmonella antibodies within hosts (all P-values > 0.164; see online appendix tables S2a and S2b). 
Disease occurs during the initial phase of infection only, but long-term persistence incurs

\section{a reproductive cost}

Salmonella Typhimurium infection dynamics in an experimentally infected group of pigeons were followed up during 66 weeks, spanning two consecutive breeding seasons (Fig. 2) and including natural stress periods (molt, breeding, introduction of new group members). Shortly after inoculation, Salmonella shedding increased drastically (Fig. 2).

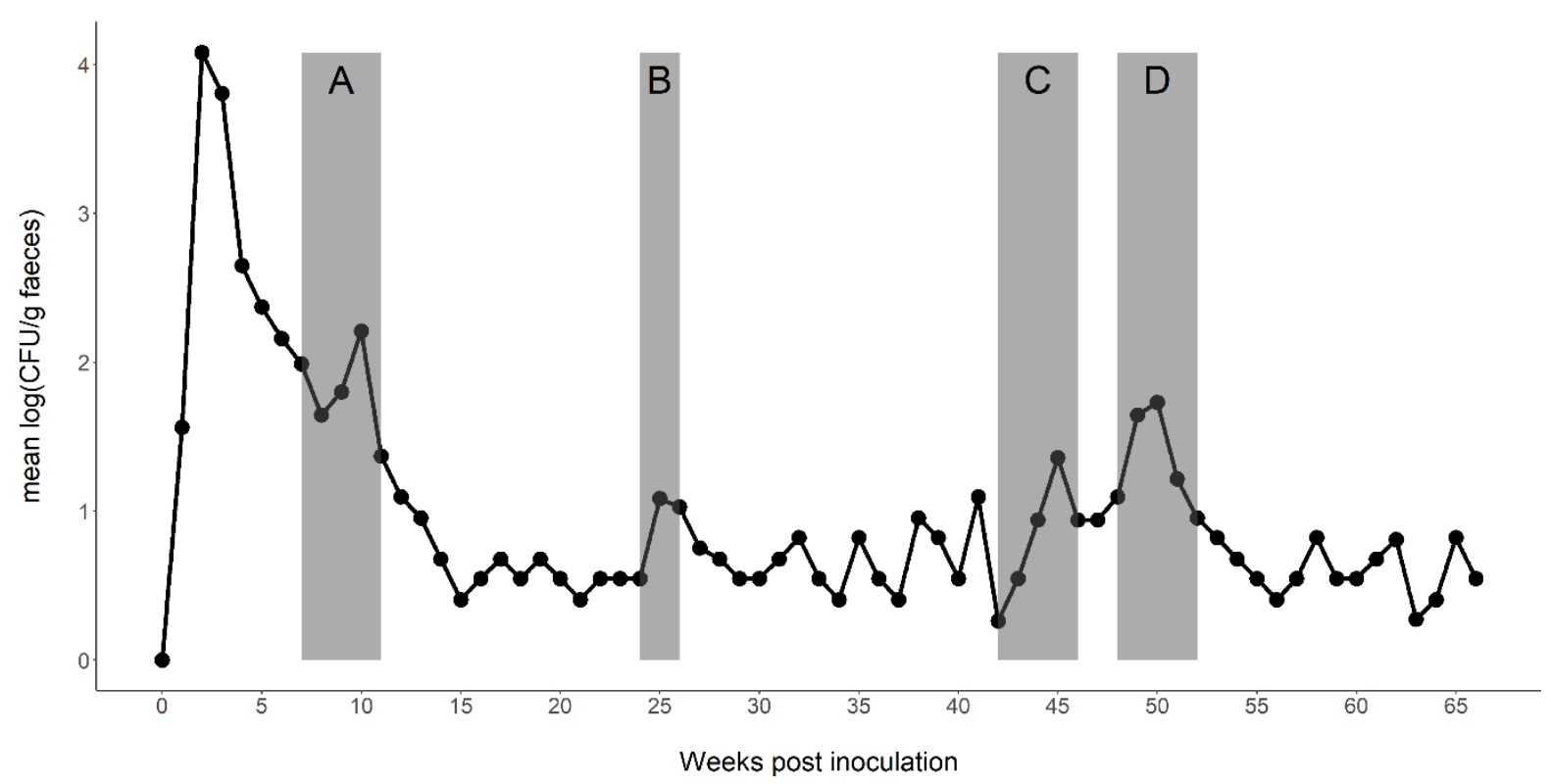

Fig 2. Temporal dynamics of faecal Salmonella shedding by experimentally infected pigeons.

Experimentally inoculated pigeons showed a significant increase in shedding during: A, breeding period 1 (Parental); B, molting; C, introduction of naïve pigeons and D, breeding period 2 (Parental + F1 generation). Grey bars indicate the duration of each stressful period.

The best fitting model (i.e. the lowest AIC-value: 17.38; table S3) obtained for describing trends in Salmonella shedding after the inoculation period had an ARIMA $(1,0,1)$ structure with non-zero mean, and included both induced stress periods and the number of pigeons present as important external regressors (P-values $<0.0001)$. This first-order autoregressive model (AR1 estimate and standard error: $-0.47 \pm 0.26)$ with simple exponential 
smoothing confirms that after inoculation, Salmonella faecal shedding declines towards a nonzero mean $(2.27 \pm 0.29, \mathrm{P}$-value $<0.0001$; i.e. Salmonella remains present in the population).

Alleged stress periods correspond to temporary increases in Salmonella shedding $(0.42 \pm 0.08$, P-value $<0.0001)$ but with a negative correlation between the number of pigeons in an aviary and Salmonella faecal shedding $(-0.04 \pm 0.01, \mathrm{P}$-value $<0.0001$; table S3).

Salmonella was shed intermittently at low levels in the faeces during the entire infected group during the 66-week experiment. Only shortly after initial and experimental inoculation of the naive founder pigeons, clinical signs of paratyphoid (e.g. diarrhea, anorexia, polydipsia) were observed. Body mass trends differed between the experimentally infected and the control group (interaction between month and treatment, P-value $<0.0001$ ), but this difference is mainly due to lower body masses of experimentally inoculated pigeons in the time periods immediately following the inoculation (experimental infection versus control group month 1: $-39.10 \pm 7.65, \mathrm{P}$-value $<0.001 ;$ month 2: $-23.65 \pm 7.70, \mathrm{P}$-value $=0.012$ ). In later months, body weights of infected birds remained lower than those of control birds (P-values > 0.05 but $<0.10)$ but only for the last month, this difference was significant $(\mathrm{P}$-value $=0.03$, of paratyphoid were noted thereafter. 


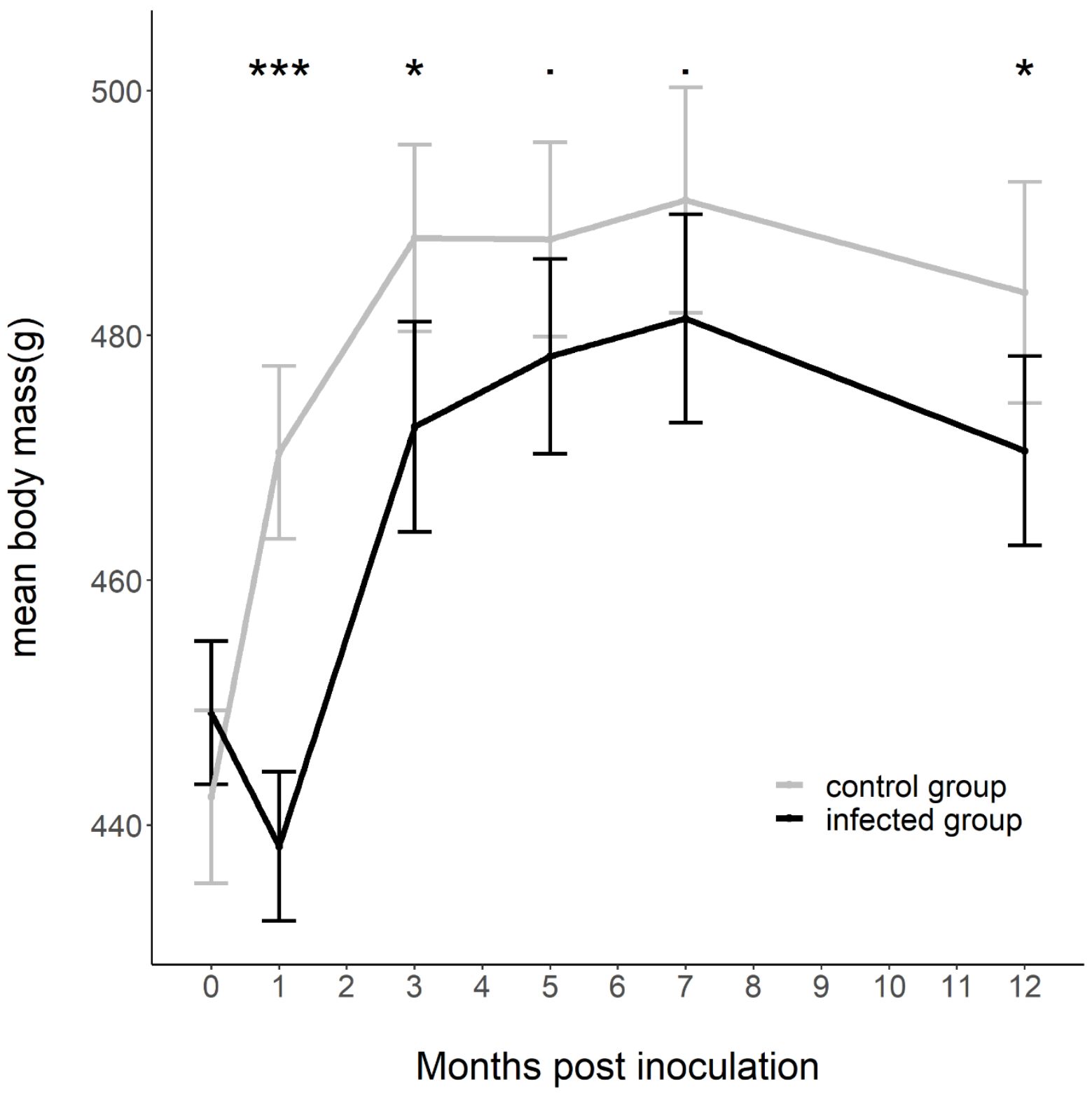

147 Fig 3. Mean body weight of the adult pigeons in the Salmonella infected vs. the negative control

148 group. The mean body weight per group is given (mean \pm se) before and after inoculation with

149 Salmonella Typhimurium varietas Copenhagen DAB69 and sham inoculation respectively.

150 Significance codes: $* * *<0.001, * *<0.01, *<0.05,0.05<.<0.10$. 

the crop did not simultaneously shed detectable numbers of Salmonella in the faeces. During the first nesting period (28 days), no Salmonella was detected in any of the cloacal swabs, but during the second breeding period, Salmonella was detected in the cloacal swabs of $53.3 \%$ (8/15) of the nestlings, with first Salmonella detections on average from day $4.6 \pm 3.6$ post hatching.

\section{Association with the host reproductive tract does not typically result in vertical pathogen}

\section{transmission but impairs host reproductive success}

20 testes. Presence of Salmonella in the pigeons' reproductive tissues was confirmed by immunohistochemical staining (Fig. S1a-b). Despite this association with the pigeons' gonads, Salmonella could not be isolated from any of the semen samples $(\mathrm{n}=121)$ collected during the whole experimental trial. Salmonella was found in $4.5 \%$ of the eggs laid (i.e. five out of 111) in the infected group. Four of these 5 eggs were found to be non-fertilized. At day of hatch, none of 35 faecal and cloacal swabs sampled from hatchlings in the infected pigeon group were positive for Salmonella during both breeding periods. We thus conclude that, despite strong association with the female reproductive tract, vertical pathogen transmission to the offspring is a rare event.

Association of Salmonella with the host reproductive tract correlated with reduced reproductive performance through negative effects on both eggs and nestlings. Salmonella

177 infected pigeons had nests characterized by lower egg masses, more shape/shell anomalies and 
a lower hatching success compared to control group pigeons. Hatchlings also had lower body masses, showed delayed fledging and gained less mass in the 28 days after fledging, -compared to the negative control group (all P-values $<0.028$, table 1; tables S5a-f). Clutch size, the number of nests with one egg only and the prevalence of infertile eggs did not differ between groups (all P-values $>0.219$, table 1; tables S5g-i). During the first breeding period, none of the 20 nestlings died due to paratyphoid in the infected group. During the second breeding period in this group, five out of 15 nestlings died of paratyphoid during the first 28 days after hatching (on average at day $16 \pm 9$ ). No clinical signs were observed among the remaining nestlings, except for 1 nestling during the second breeding period which presented depression and right elbow joint arthritis. Breeding phenology was similar too, as no differences were found in the onset of nesting, nest building and mating behavior, nor in laying dates (P-value $=0.889$, table $1 ;$ table $\mathrm{S} 5 \mathrm{j})$.

Table 1. Reproductive parameters measured during breeding periods. All results are given as total number or average number $\pm \mathrm{SD}$.

\begin{tabular}{llcc}
\hline Parameter & Negative control group & Infected group & p-value \\
\hline Clutch size & $9.44 \pm 0.53$ & $8.54 \pm 2.3$ & 0.537 \\
Shape/shell anomalies & 0 out of 86 eggs & 12 out of 111 eggs & $\mathbf{0 . 0 1 1}$ \\
Number of 1 egg nests & 10 out of 28 nests & 20 out of 36 nests & 0.219 \\
Egg mass (g) & $19.36 \pm 2.21$ & $18.11 \pm 2.28$ & $\mathbf{0 . 0 0 0 9}$ \\
Salmonella presence & 0 out of 86 eggs & 5 out of 111 eggs & n.a \\
Infertile eggs & 15 out of 71 eggs & 26 out of 92 eggs & 0.299 \\
Laying date & $7.25 \pm 0.45$ & $7.28 \pm 0.57$ & 0.889 \\
Hatching success & $88.63 \%$ & $75.9 \%$ & $\mathbf{0 . 0 1 5}$
\end{tabular}


Hatchling mass $(\mathrm{g})$

Mass gain over 28-day period (g)

Nestling mortality (up to day 28)

Fledging age (days)
$17.27 \pm 3.67$

$378 \pm 46$

$96.4 \%$

$28.04 \pm 0.51$
$15.41 \pm 2.19$

0.015

$328 \pm 61$

0.005

0.028

$74.3 \%$

$30.03 \pm 1.28$

\section{Acquired population immunity protects against clinical disease but not infection}

The host immune response to Salmonella infection was evidenced by a pronounced

humoral response in the adult pigeons: Enzyme Linked ImmunoSorbent Assay (ELISA) results

revealed seroconversion of the experimentally infected pigeons from four weeks after inoculation, whereas the control group remained negative. Infected pigeons seroconverted and remained seropositive until the end of the 66-week experiment (P-value $=0.0010$; Fig. S2;

Table S6a). The offspring of these pigeons was characterized by significantly higher levels of maternal anti-Salmonella antibodies (IgY) in their blood at birth as compared to the control group $(\mathrm{P}$-value $=0.0002)$. While still significantly different, these antibody titers decreased over a 14-day period (day 0 versus day 14: $\mathrm{P}$-value $=0.009$; day 14 versus day 28 : P-value $=$

0.177; Tables S6b-c; Fig S3). After an experimental oral challenge with Salmonella, agematched (5-6 months old) pigeons from the infected group showed a significantly better faecal consistency (P-value=0.0001, Table S6d), shed lower amounts of faecal Salmonella $(\mathrm{P}=$ tended to be less strongly affected than those from the control group, but these differences failed to reach statistical significance. After the experimental oral challenge with Salmonella, pigeons from the infected group did not have more severe organ lesions $(-0.083 \pm 0.116, \mathrm{P}-$ 
seroconvert slower than the juveniles born in the infected pigeon group $(-0.670 \pm 0.519, \mathrm{P}$-value $=0.208$; Table S6h, Fig S4). Pigeons from the infected group had similar Salmonella organ loads $(-0.244 \pm 0.808, \mathrm{P}=0.767$; table $\mathrm{S} 6 \mathrm{i})$ as pigeons from the control group. Pigeons from the infected group were characterized by higher body masses compared to control group pigeons (53.5 $\pm 22.3 \mathrm{~g}, \mathrm{P}=0.031$, table $\mathrm{S} 6 \mathrm{j}$ ), but both groups exhibited a similar body mass trend (decline in mass through time, $-1.844 \pm 7.148 . \mathrm{P}=0.798$; table S6k).

\section{Stress periods result in flare-ups of infection}

The two breeding periods, moult and the introduction of five naive animals in the group coincided with marked flare-ups of the Salmonella infection, as evidenced by significantly increased faecal Salmonella shedding (P- value $<0.0001$, see ARIMA model above, Fig. 2;

Table S3). This higher faecal Salmonella excretion was, however, not accompanied by the newly-introduced pigeons which started to excrete viable bacteria within on average 8 days after introduction in the positive group, though without showing any signs of illness.

To establish a causal relationship between stress and flare-up of infection, pigeons were housed individually. Individual housing resulted in significantly increased faecal Salmonella shedding (P-value < 0.0001; Fig 4A, table S7a), and increased the variance in corticosterone levels in the individually housed pigeons compared to the control group (control group: no change in variance, F-test $\mathrm{P}$-value $=0.584$, experimental group: increase in variance, F-test $\mathrm{P}$ value $<0.001$, table S7b). Mean plasma corticosterone levels increased as well in individually housed pigeons, although this increase failed to reach statistical significance compared to 


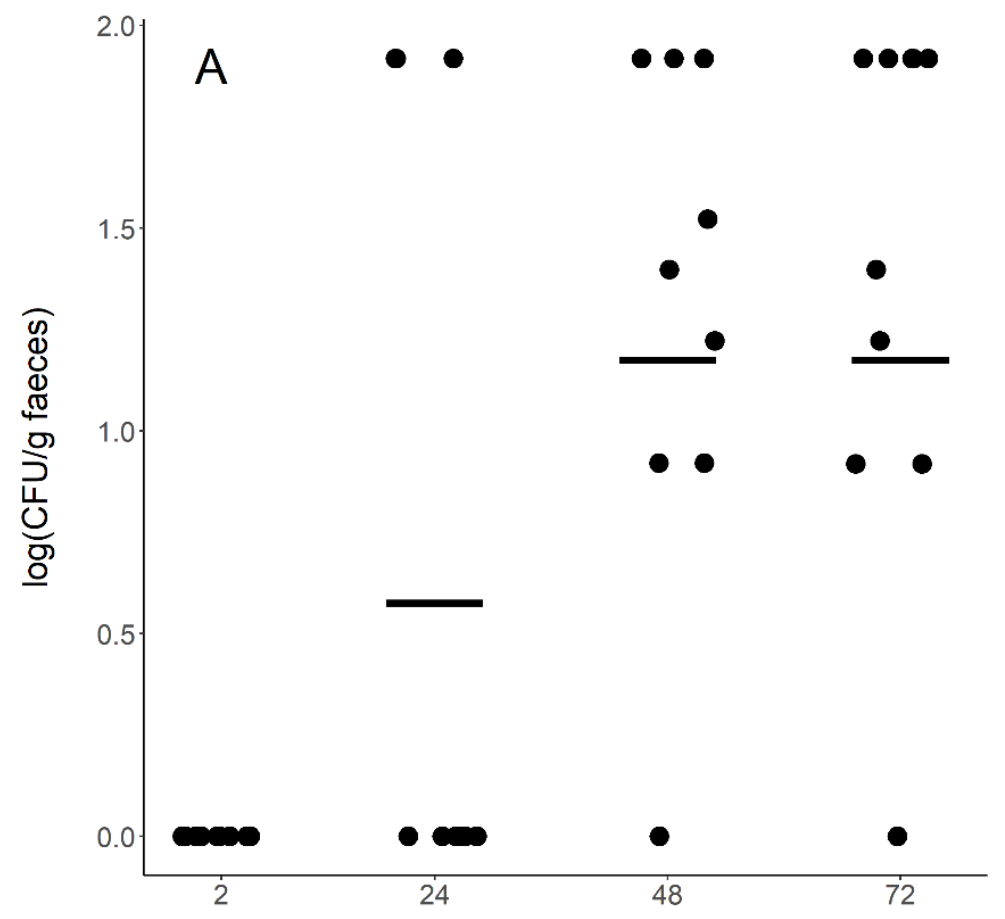

Time (h) after individual housing

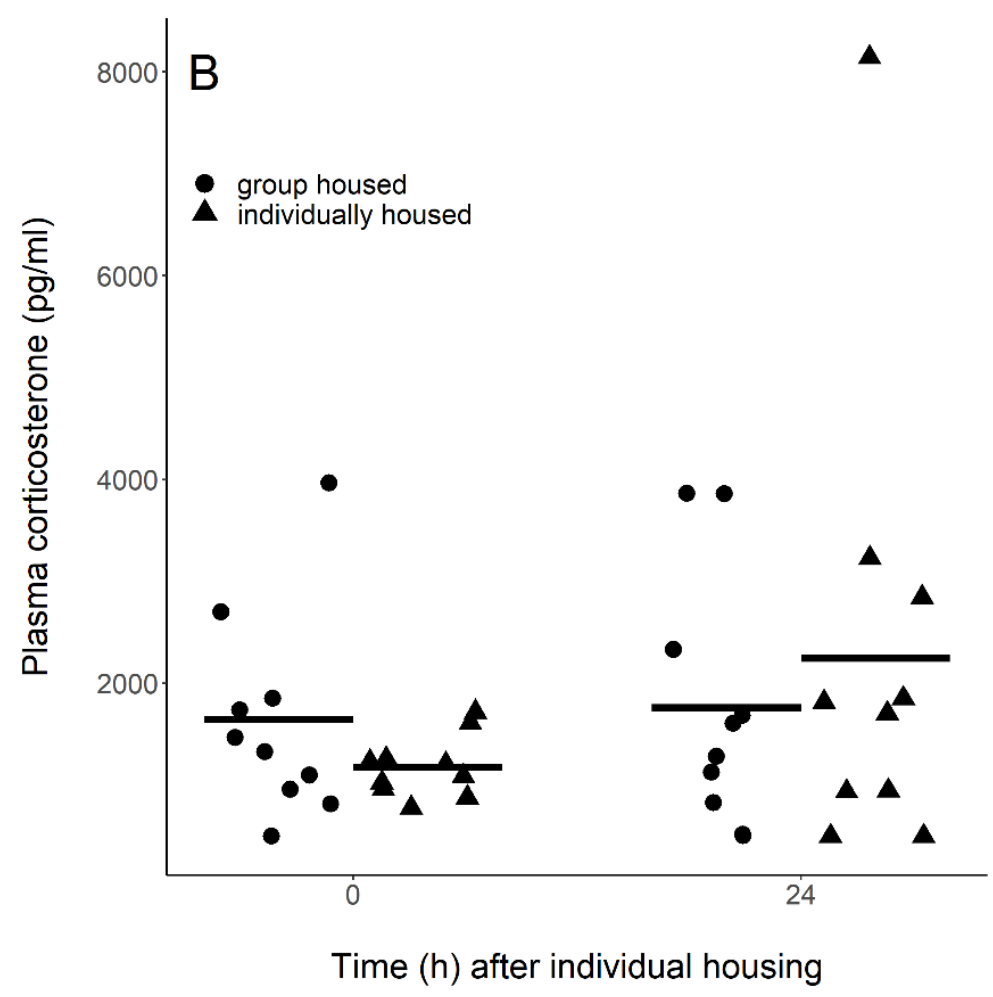

239 Fig 4. Faecal Salmonella shedding (A) and plasma corticosterone levels (B) in pigeons, housed 240 individually or in group. Each symbol represents one pigeon and the horizontal bar indicates 241 the mean value. 


\section{Discussion}

\section{Impaired host condition and reproduction offset by population immunity in a context of}

\section{pathogen endemism}

Successful long-term host-pathogen co-existence entails trade-offs and benefits for

both host and pathogen. From the perspective of a host-restricted pathogen, the limited

availability of potential hosts supposedly is outweighed by a pathogen competitive advantage

restricted pathogen in the host population comes with a cost for host condition and reproductive success but also with a clear long-term benefit for host disease resistance. Salmonella affected body weight and thus body condition in infected pigeons. While more severe during early establishment of infection, this effect persisted at least one year after pathogen introduction. caeruleus) [18,19], schistosome infections in snails [20] and Toxoplasma gondii infections in mice [21]. Impaired reproduction and weight gain could be explained by increased host investment (e.g. acquired immunity) in controlling pathogen burden [18,21] and by the

be considered a trade-off for both the pathogen (reduction of the number and condition of suitable hosts) and the host (potential negative impact on population persistence). The host investment in immunity confers protection against paratyphoid disease at population level. The presence of high titers of antibodies in nestlings at day of hatching combined with exposure to 
of serum antibody levels by 14 days of age coincided with elevated mortality in older nestlings, be widely present in populations of feral pigeons, population immunity is likely to offer a distinct advantage. At population level, the protection against clinical disease and population decline at least partly compensates condition and reproductive costs associated with hostpathogen co-existence.

\section{Exploiting host characteristics for pathogen transmission and persistence} in a host population [27]. We here demonstrate that Salmonella exploits the host specific the stress associated with the breeding period resulted in a significant Salmonella recrudescence (see further), shedding in the crop may be the mere result of the overall increased Salmonella burden in the parent pigeon. This crop-feeding route of pathogen transmission is a pseudo-vertical transmission [28], allowing fast and efficient transmission of the pathogen to the parents' offspring without the risk of infertile eggs or embryo mortality. Indeed, despite close association with the host gonads, we could not find any evidence of true vertical transmission (incorporation of Salmonella in the egg, resulting in an infected hatchling). The association of Salmonella with the pigeons' gonads thus contributes to pathogen persistence in the host, but not significantly to pathogen transmission. Persistence in the pigeon gonads may be explained by the specific niche Salmonella occupies here. Immunohistochemistry showed 
persistence through their relative inaccessibility for the host's immune system [29,30]. Despite Salmonella being considered a chiefly intracellular pathogen, residing in the host macrophages $[1,14]$, extracellular persistence in niches poorly accessible to the immune system provides evidence for multiple pathogen strategies [31].

\section{Host stress promotes pathogen dispersal}

Natural stress periods such as breeding, moult and social interactions (introduction of new birds to the group) were shown to temporarily increase Salmonella shedding in the pigeons with no discernible impact on bird health. Stress is proven to be a trigger for re-excretion of a pathogen by carrier and corticosteroids have recently been proven to drive Salmonella recrudescence [32-34]. Such temporary and corticosteroid associated recrudescence of Salmonella infection in the presence of novel hosts (newborn nestlings or naive newcomers) is likely to contribute to pathogen maintenance within a host population.

\section{Conclusion}

We have demonstrated that Salmonella Typhimurium exploits host characteristics associated with reproduction and stress to establish and maintain endemism in the pigeon population. This entails a reproductive cost for the hosts, which is outweighed by the development of immunity that protects the birds against pathogen-induced mortality and results in a stable pathogen reservoir.

. 
Salmonella Typhimurium faecal shedding and seroprevalence in feral pigeons screening cloacal swabs and serum from pigeons in four populations ((Brussels $(50,84667 / 4,35472 ; \mathrm{n}=30)$, Antwerp $(51,22139 / 4,39722 ; \mathrm{n}=35)$, Bruges $(51,20944 / 3,22528$; $\mathrm{n}=39)$ and Louvain $(50,8775 / 4,70444 ; \mathrm{n}=29))$ for the presence of Salmonella Typhimurium and antibodies against Salmonella Typhimurium respectively. A clinical examination was done showing signs of ill health were scored 1 . A body condition score, adapted from Gregory and Robins (1998) and Møller et al. [36], ranging from 1 to 5 was assigned, based on the bird's general appearance, breast muscle size and crista sternalis/breast muscle ratio; 1: skinny, muscle atrophy, clearly noticeable crista sternalis; 2: underdeveloped breast muscles, crista sternalis noticeable; 3: normal muscle size, crista sternalis palpable; 4: well fed, firm, big breast muscles, crista sternalis palpable but not clearly noticeable; 5: obese, big breast muscles, presence of subcutaneous fat behind the sternum. Bacteriological analysis of cloaca swabs and an ELISA on serum samples were performed as described below. For the feral pigeons, it was necessary to first determine the Salmonella serotypes present in the faeces. Therefore, tests were performed on possible ('pink') colonies, which were confirmed to be Salmonella based on their biochemical characteristics: glucose fermentation, absence of lactose fermentation, $\mathrm{H} 2 \mathrm{~S}$ production, lysine decarboxylation positive and absence of urease activity. Subsequently they were serotyped by slide agglutination, targeting the somatic antigens (Pro Lab 
Experimental animals

Forty clinically healthy one-month-old pigeons were obtained from a captive breeding

colony free of Salmonella. The animals were negative for the presence of Salmonella in their

faeces on multiple samplings at 1-week intervals as well as for agglutinating antibodies and anti-Salmonella IgY antibodies (ELISA) in their blood (see below). Sex was determined using a polymerase chain reaction targeting the CHD genes as described by Griffiths et al. (1998). They were group-housed in an aviary with a $12 \mathrm{~h}$ photoperiod and fed a commercial seed-based diet ad libitum. All the experiments were carried out with the approval of the ethical committee of the Faculty of Veterinary Medicine, Ghent University (EC2013/137; EC2014/96; EC2015/01).

\section{Experimental Design}

Two pigeon groups (similar in age and sex) were formed from the same parental population. In the first group $(\mathrm{n}=20)$, an endemic Salmonella Typhimurium infection was established and followed during 66 weeks. The second group $(\mathrm{n}=20)$ was not infected and served as negative control, providing baseline data for clinical signs and reproductive parameters. Mechanisms and trade-offs of Salmonella endemism were examined by 1) determining Salmonella infection dynamics with focus on identifying routes of transmission, both horizontal (faecal shedding, crop feeding) and vertical (egg and/or semen contamination), 2) quantifying the effect of a Salmonella infection on pigeon health and reproductive parameters 3), estimating the role of population immunity in protection against clinical 

of 20 pigeons each: (i) pigeons inoculated with Salmonella, and (ii) pigeons sham-inoculated. Each pigeon of the first group was inoculated in the crop with $1 \times 10^{8}$ Colony Forming Units (CFU) of the bacterial suspension in $1 \mathrm{~mL}$ of inoculum. Birds from the second group were sham-inoculated with LB broth and served as a negative control group. After inoculation, the pigeons, their eggs and their offspring were followed for 15 months (including two breeding seasons) to quantify infection and disease dynamics. Birds were examined daily for the presence of paratyphoid symptoms (anorexia, polydipsia/polyuria, diarrhea, weight loss...) and faecal shedding of the Salmonella strain. Salmonella shedding in the crop of crop-feeding adults was assessed daily by collecting crop swabs from both pigeon groups. Faecal consistency was scored daily as described by Pasmans et al. (2008): 0, normal faeces; 1, faeces not well formed; 2 , watery faeces; 3 , severe diarrhea; 4 , hematochezia; 5 , absence of faecal production combined with anorexia.

The numbers of Salmonella CFU per gram of matrix were determined by plating tenfold serial dilutions on Brilliant Green Agar (BGA) plates (LabM, Lancashire, UK). If negative after direct plating, the samples were pre-enriched overnight in buffered peptone water (Oxoid, Basingstoke, UK) at $37^{\circ} \mathrm{C}$ and then enriched in tetrathionate brilliant green broth (Merck $\mathrm{KGaG}$, Darmstadt, Germany) at $37^{\circ} \mathrm{C}$. Therefore, within the infected group, faeces was collected on a daily base to obtain the average daily number of CFU for the entire group and subsequently the average weekly number of CFU. Cloacal swabs and crop swabs were plated on BGA agar and investigated for the presence of Salmonella as described above.

At the end of the experiment, all Salmonella-infected pigeons were humanely euthanized with an intravenous injection of sodium pentobarbital $(100 \mathrm{mg} / \mathrm{kg}$, Natrium 
Pentobarbital 20\%, KELA, Belgium) and necropsied. The organs (intestines excluded) were scored for the presence of lesions by the same person using the following system: 0 , no macroscopic lesions; 1, organ enlargement; 2, presence of small granuloma's of $<3 \mathrm{~mm} ; 3$, presence of granuloma's of 3-6 mm; 4, presence of granuloma's of 7-9 $\mathrm{mm} ; 5$, presence of granuloma's of $>9 \mathrm{~mm}$. The intestines were scored using the following system: 0 , no macroscopic lesions; 1 , serosal congestion; 2, abnormal content; 3, hemorrhagic content; 4, presence of granuloma's of $<3 \mathrm{~mm} ; 5$, presence of granuloma's of $>3 \mathrm{~mm}$. Tissues were homogenized and the number of CFU of Salmonella per g tissue was determined as described above.

Dynamics of circulating antibodies in pigeons with endemic Salmonella infection

We used serum anti-Salmonella antibodies dynamics as proxy for the acquired immunity response against the Salmonella strain in the pigeon groups. To detect antiSalmonella antibodies, a specific eELISA was developed. This ELISA was home-made according to Leyman et al. (2011) with some minor modifications. ELISA plates (F96 maxisorp Nunc-immuno plates, Nunc, Roskilde, Denmark) were coated with $140 \mu \mathrm{L}$ of a suspension containing formalin-inactivated Salmonella Typhimurium varietas Copenhagen strain DAB69 bacteria diluted in coating buffer to an optical density (OD) of $660 \mathrm{~nm}$, measured with a spectrophotometer (Ultraspec III ${ }^{\circledR)}$. Serum was diluted 1/1000 and added to the wells $(100 \mu \mathrm{L})$. Conjugate consisted of a 1/1000 dilution of a goat anti-bird IgY antibody (Alpha Diagnostics International, San Antonio, Texas, USA). All samples were run in triplicate; 2 positive and 2 negative controls were added to each plate. The washing steps in this protocol density was measured using a Multiskan MS Reader (Labsystems Oy, Helsinki, Finland) with 417 the Ascent Software, version 2.6. Using this ELISA, blood samples (0.5 mL), collected at 2- 
month intervals were examined for the presence of anti-Salmonella antibodies in all pigeons of both the infected and non-infected group during the whole experiment.

\section{Protection of pigeons from a group with endemic Salmonella infection against clinical}

\section{salmonellosis}

To assess to what extent pigeons born in the endemically infected group were protected against a subsequent Salmonella infection and to what extent the presence of anti-Salmonella antibodies correlated with protection, we randomly removed eight age- and sex-matched pigeons born in the negative control group and eight born in the infected group from their groups and inoculated them with the Salmonella strain. The pigeons of between 5-6 months of age were individually housed and a blood sample $(0.5 \mathrm{~mL})$ was taken prior inoculation to check for the presence of anti-Salmonella IgY antibodies. Each pigeon was inoculated in the crop with $1 \times 10^{3} \mathrm{CFU}$ Salmonella Typhimurium PT99 DAB69 in $1 \mathrm{~mL}$ of inoculum. The numbers of Salmonella CFU per gram of faeces were determined and the birds were followed up clinically as described above. At day 14 post inoculation, a blood sample $(0.5 \mathrm{~mL})$ was taken and the pigeons were humanely killed. Internal organs were collected and processed for Salmonella quantification as described above.

\section{Reproductive cost of an endemic Salmonella infection}

Two months after inoculation with Salmonella, the dark/light cycle was gradually adjusted to a daily $16 \mathrm{~h}$ photoperiod. Both groups of 20 pigeons each were housed in an indoor aviary $(5 \times 2.5 \times 2.5 \mathrm{~m})$, given water and feed ad libitum and were provided with nesting sites and given the opportunity to make nests and start breeding to study the effect of an endemic pathogen on the host's reproductive success. We collected data in both groups with regard to a set of parameters, related to reproduction, to estimate the impact of an endemic Salmonella 
infection on host reproduction. The time required to proceed to nest building, the time until the

444 first egg was laid and the number of eggs laid was noted for pigeons in both groups. After laying, the eggs were weighed to the nearest $0.01 \mathrm{~g}$ and measured (length and width) using a caliper to the nearest $0.1 \mathrm{~mm}$ and given a unique number. To assess whether Salmonella is incorporated in the pigeons' eggs (vertical transmission), the first clutch of eggs was examined for the presence of Salmonella. Therefore a swab was taken from the egg shell before egg surface decontamination. Subsequently, the egg was opened and the egg yolk and albumen were removed followed by egg shell and membrane homogenization. Each egg matrix was examined for the presence of Salmonella using the above described method. To assess the potential role of semen in Salmonella transmission between birds, semen was collected 1-2 times a week from the male pigeons in both groups using a lumbo-sacral and cloacal region massage technique $[39,40]$. Semen samples were examined for the presence of Salmonella as described above.

The pigeons were allowed to incubate the second clutch of eggs. The following parameters were noted: total number of eggs per group, clutch size and egg mass. Unfertilized eggs, determined by egg candling every two days, were processed for Salmonella culture as described above. At hatching, nestlings were weighed and a blood sample ( $20 \mu \mathrm{L})$ was taken from the vena jugularis to determine the presence of maternal IgY using ELISA. The number of unhatched eggs was noted and again these eggs were processed for Salmonella culture as described above. From then on, the young nestlings were weighed daily until the age of 28 days (fledging age). Blood was taken at 14 and 28 days of age $(0.5 \mathrm{~mL})$ from the vena ulnaris superficialis. Cloacal swabs and crop swabs were taken daily from day 1 until fledging and checked for the presence of Salmonella. Additionally, crop swabs were taken from the cropfeeding parents. The offspring was further kept in the parental group. One year later, the F1 
generation of pigeons was also allowed to breed and raise young. All procedures were repeated as described above.

\section{Role of stress during Salmonella endemism in pigeons}

Periodic stress may be important in maintaining endemic infections through its potential to induce infection flare-ups [41]. Known stress periods in our experiments were the introduction of new animals, the molting period and the breeding period $[32,42-44]$, and we tested whether exposing pigeons to such stressors influenced the Salmonella numbers shed in their faeces.

To test for a causal link between stress and Salmonella shedding in faeces, we used single housing as a stressor (often used in stress-related research, e.g. see Baker et al., 2019; Dunn et al., 2015) and determined faecal shedding of Salmonella and corticosterone levels in the blood. Ten pigeons from the infected group (chosen ad random, equal sex ratio) were individually housed. A blood sample $(0.5 \mathrm{~mL})$ was taken from each pigeon prior to individual housing and $24 \mathrm{~h}$ later, this within $30 \mathrm{sec}$ after capture to avoid any other influence on corticosterone levels. After individual housing, faecal samples from each pigeon were collected (within 2 and after $24 \mathrm{~h}$, and after 48 and $72 \mathrm{~h}$ ) for bacteriological titration. As a control, blood samples $(0.5 \mathrm{~mL})$ were collected at similar time points from pigeons that stayed in their group. Faecal samples were processed as described before to quantify the number of Salmonella bacteria.

To evaluate the effect of the introduction of new animals as a stressor on the group, in a second experiment, we examined the fate of naive pigeons upon introduction in the Salmonella positive group. For this purpose, 5 randomly selected pigeons ( 3 males, 2 females), born in the negative control group, were introduced in the infected pigeon group. Cloacal swabs 
were taken daily and processed as described above. After 1 month, a blood sample was taken and IgY antibodies were assessed using the Salmonella specific ELISA as described above.

\section{Corticosterone analysis}

\section{Sample pre-treatment and LC-MS/MS analysis}

To $50 \mu \mathrm{L}$ of plasma we added $50 \mu \mathrm{L}$ of the internal standard (IS) working solution (corticosterone-d8, $10 \mathrm{ng} \mathrm{mL}^{-1}$ in methanol) and $400 \mu \mathrm{L}$ of liquid chromatography-mass spectrometry (LC-MS) grade water, followed by a vortex mixing (15 sec) and equilibration (5 min, room temperature) step. After the addition of $3 \mathrm{~mL}$ of diethylether, the samples were extracted for $20 \mathrm{~min}$ on a rotary apparatus. The samples were centrifuged for $10 \mathrm{~min}$ at 4750 $\mathrm{rpm}$ and $4^{\circ} \mathrm{C}$. The supernatant was transferred to another tube and evaporated to dryness using a gentle stream of nitrogen $\left(\mathrm{N} 2 ; \sim 40^{\circ} \mathrm{C}\right)$. The dry residue was reconstituted in $75 \mu \mathrm{L}$ of LCMS grade methanol and vortexed for $15 \mathrm{sec}$, followed by the addition of $75 \mu \mathrm{L}$ of LC-MS grade water. After vortexing, the sample was passed through a $0.22 \mu \mathrm{m}$ Millex ${ }^{\circledR}$ Nylon syringe filter and transferred to an autosampler vial. A $10 \mu \mathrm{L}$ aliquot was injected onto the liquid chromatography-tandem mass spectrometry (LC-MS/MS) instrument. The LC-MS/MS system consisted of an Acquity H-Class Quaternary Solvent Manager and Flow-Through-Needle Sample Manager with temperature controlled tray and column oven in combination with a Xevo TQ-S ${ }^{\circledR}$ MS/MS system, equipped with an electrospray ionization (ESI) probe operating in the positive mode (all from Waters, Zellik, Belgium). More details about the LC-MS/MS method can be found in De Baere et al. (2015) (47).

Quantification of corticosterone in pigeon plasma was performed using matrix-matched calibration curves. The calibrator samples were prepared by spiking $50 \mu \mathrm{L}$ aliquots of pigeon plasma with corticosterone levels of $0.0,0.5,1.0,2.0,5.0,10.0$ and $20 \mathrm{ng} \mathrm{mL}^{-1}$. The standard working solutions of corticosterone and the IS were directly applied onto the samples, followed 
by a vortex mixing step. After 5 min of equilibration, the sample preparation procedure was performed as described above. The basal concentration of corticosterone in the plasma samples used for the preparation of the calibration curve was determined in order to correct the corticosterone concentrations in the study samples. Limits of quantification (LOQ) and detection (LOD) were $0.5 \mathrm{ng} \mathrm{mL}^{-1}$ and $0.2 \mathrm{ng} \mathrm{mL}^{-1}$ respectively.

\section{Immunohistochemistry}

The gonads (ovary, oviduct and testes) were fixed in $4 \%$ phosphate buffered formaldehyde and embedded in paraffin for routine light microscopy. The immunohistochemical staining protocol to specifically stain the Salmonella bacteria was done as described by Morrison et al. (2012) and Van Parys et al. (2010) with some modifications (48, 49). In short, sections of $5 \mu \mathrm{m}$ were cut followed by deparaffinization, hydration and antigen retrieval in citrate buffer ( $\mathrm{pH}$ 6.0) using a microwave oven. Slides were incubated with a $3 \% \mathrm{H}_{2} \mathrm{O}_{2}$ in methanol solution (5 min) and $30 \%$ goat serum (30 min) to block endogenous peroxidase activity and non-specific reactions, respectively. This was followed by an incubation step with a Polyclonal rabbit anti-Salmonella $\mathrm{O} 4$ antibody, targeting the $\mathrm{O} 4$ somatic antigen (1/1000; Pro Lab Diagnostics, Bromborough, UK) for 30 min. A biotinylated goat antirabbit IgG antibody (1/500; DakoCytomation, Glostrup, Denmark) served as secondary antibody. After rinsing, the sections were incubated with a streptavidin-biotin-HRP complex (DakoCytomation) and the brown color was developed with diaminobenzidine tetrahydrochloride (DAB, Dako) and $\mathrm{H}_{2} \mathrm{O}_{2}$. Counterstaining was done using hematoxylin before dehydration and coverslip placement. All incubation steps were done using a Dako Autostainer apparatus.

\section{Statistical analyses}


Salmonella infections are endemic in feral pigeon populations deriving a Bayesian estimate of true prevalence from apparent prevalence obtained by testing individual samples, using a sensitivity value of at least 0.90 and a specificity value of at least 0.99 [51]. To test whether Salmonella presence was related to pigeon health status or body antibodies and pigeon sex and age (and their two-way interactions) were included as fixed effects. Health status was modelled using a binomial model, for health score a Gaussian error distribution was used. We adopted a frequentist approach whereby full models (i.e. models

excluding the variable with the highest $\mathrm{p}$-value until only $\mathrm{p}<0.05$ predictors remained.

Statistics and P-values mentioned in the text and tables are from the minimal model (all significant terms included), whereas statistics and P-values of non-significant terms were obtained by fitting each non-significant term separately into the minimal model.

Endemism noticed in the wild can be experimentally replicated, and is sustained mainly

by horizontal transmission despite marked pathogen association with the host

\section{reproductive tract}

To test whether Salmonella Typhimurium becomes endemic when introduced into a 
the colony's faeces. From an epidemiological perspective, CFU density in a given week will

567 likely be correlated with the previous week, and measurements thus do not represent independent data and should be treated as a time-series. We therefore opted to apply an ARIMA (autoregressive integrated moving average) model to analyze temporal dynamics of faecal Salmonella shedding. Models were fitted using the 'auto.arima' function of the R library 'forecast' [53], using AIC values as criterion for model selection. Designated periodic stress events (i.e. breeding periods, molting periods and introductions of new individuals, see methods) and the total number of pigeons in the colony were included as covariates. Variable coefficients were divided by their standard errors to obtain the z-statistics, which were then used to calculate the P-values reported.

To compare trends in body mass of experimental versus control group pigeons, a linear

mixed model was used. Body mass was specified as a dependent variable and time period effects. Individual pigeon identity was always included as a random effect. Model were run with a Gaussian error distribution. In addition to testing overall trends throughout the experiment, we verified at which time periods significant differences between experimental and control group arose using the glht function of R library 'multcomp' [54], to obtain P-values corrected for multiple testing.

\section{Salmonella endemism impairs host reproduction but does not affect adult pigeon health}

resulted in impaired reproduction, differences in reproductive parameters between the 
(experimental versus control) and their interaction as fixed effects. Models were run with either binomial or Gaussian error distribution as appropriate, and variables were log transformed when needed to obtain normality of residuals (i.e. Shapiro-Wilk $\geq 0.95$ ).

\section{Endemism coincides with population immunity offering protection against clinical}

\section{disease but not infection}

To test whether the experimental inoculation of naïve pigeons born in the negative control group resulted in an increase in clinical symptoms, the occurrence and/or severity of several parameters for disease in the experimental (pigeons born in the infected group) versus the (naïve) control group of pigeons was assessed using linear mixed models. These parameters (i.e. faecal Salmonella count, body mass, faecal consistency scores, diverse morphological anomalies, organ lesions, Salmonella presence in organs and antibody titers; see above) were collected either daily, weekly or at the end of the experiment after euthanasia. Differences between experimental and control groups were tested for by specifying any of the aforementioned symptoms as dependent variable and time period (daily or weekly), treatment (experimental versus control) and their interaction as fixed effects. For organ lesions at euthanasia, only treatment was included as fixed effect. Individual pigeon identity was always included as a random effect. Models were run with either binomial or Gaussian error distribution as appropriate, and variables were log transformed when needed to obtain normality of residuals.

\section{Stress periods result in flare-ups of infection}

Linear models were used to test whether individual housing (stressor) of endemically infected pigeons resulted in an increase in faecal Salmonella shedding accompanied by an increase in plasma corticosterone levels. To test whether housing pigeons individually 

increased faecal Salmonella shedding, amount of faecal Salmonella was specified as the

617 dependent variable and time as fixed effect (time after individual housing). Differences in mean

618 plasma corticosterone levels between the control and the experimental group were tested by 619 specifying time period (time after individual housing), treatment (experimental versus control) and their interaction as fixed effects. Models were run with a Gaussian error distribution. Ftests were used to test whether the individual housing stress experiment affected the variance in plasma corticosterone in the control versus the experimental group. 


\section{Acknowledgements}

642 The authors would like to thank C. Puttevils, D. Ameye, S. Loomans and S. De Baere for their

643 excellent technical assistance. We are grateful to the authorities of Brussels, Antwerp, Bruges

644 and Louvain for providing feral pigeons. The authors declare no competing financial interest.

645

646

647

648

649

650

651

652

653

654

655

656

657

658

659

660

661

662

663

664

665 


\section{References}

667

668

669

670

671

672

673

674

675

676

677

678

679

680

681

682

683

684

685

686

687

688

689

690

1. Ruby T, Mclaughlin L, Gopinath S, Monack D. Salmonella’s long-term relationship with its host. FEMS Microbiol Rev. 2012; 36: 600-615. doi:10.1111/j.15746976.2012.00332.x

2. Porta M. A Dictionary of Epidemiology. $5^{\text {th }}$ edition. Oxford University Press; 2008.

3. Schukken YH, Green L, Medley G. Epidemiology of Endemic Infectious Diseases: Accept Endemicity or Eliminate? American Association of Bovine Practitioners Proceedings of the Annual Conference, (40th), 99-107. https://doi.org/10.21423/aabppro20074526

4. Truscott JE, Ferguson NM. Transmission dynamics and mechanisms of endemicity of scrapie in the UK sheep population. Epidemiol Infect. 2009;137: 762-774. doi:10.1017/S0950268808001052

5. Maillard JC, Van KP, Nguyen T, Van TN, Berthouly C, Libeau G, et al. Examples of probable host-pathogen co-adaptation/co-evolution in isolated farmed animal populations in the mountainous regions of north Vietnam. Annals of the New York Academy of Sciences. 2008; 1149: 259-262. doi:10.1196/annals.1428.086

6. Kallio ER, Voutilainen L, Vapalahti O, Vaheri A, Henttonen H, Koskela E, et al. Endemic hantavirus infection impairs the winter survival of its rodent host. Ecology. 2007; 88: 1911-1916. doi:10.1890/06-1620.1

7. Woolhouse MEJ, Webster JP, Domingo E, Charlesworth B, Levin BR. Biological and biomedical implications of the co-evolution of pathogens and their hosts. Nature Genetics. 2002; 32: 569-577. doi:10.1038/ng1202-569

8. Benskin CMWH, Wilson K, Jones K, Hartley IR. Bacterial pathogens in wild birds: A review of the frequency and effects of infection. Biol Rev. 2009; 84: 349-373. 
doi:10.1111/j.1469-185X.2008.00076.x

692

693

694

695

696

697

698

699

700

701

702

703

704

705

706

707

708

709

710

711

712

713

714

715

9. Longini, Jr. IM, Yunus M, Zaman K, Siddique AK, Sack RB, Nizam A. Epidemic and Endemic Cholera Trends over a 33-Year Period in Bangladesh. J Infect Dis. 2002;186: 246-251. doi:10.1086/341206

10. Xiao Y, Bowers RG, Clancy D, French NP. Dynamics of infection with multiple transmission mechanisms in unmanaged/managed animal populations. Theor Popul Biol. 2007; 71: 408-423. doi:10.1016/j.tpb.2007.02.003

11. Ward MP, Cowled BD, Galea F, Garner MG, Laffan SW, Marsh I, et al. Salmonella infection in a remote, isolated wild pig population. Vet Microbiol. 2013;162: 921-929. doi:10.1016/j.vetmic.2012.11.036

12. Nielsen LR. Within-herd prevalence of Salmonella Dublin in endemically infected dairy herds. Epidemiol Infect. 2013;141: 2074-2082.

doi:10.1017/S0950268812003007

13. Wain J, Hendriksen RS, Mikoleit ML, Keddy KH, Ochiai RL. Typhoid fever. The Lancet. 2015; 385: 1136-1145. doi:10.1016/S0140-6736(13)62708-7

14. Pasmans F, Van Immerseel F, Heyndrickx M, Martel A, Godard C, Wildemauwe C, et al. Host adaptation of pigeon isolates of Salmonella enterica subsp. enterica serovar Typhimurium variant Copenhagen phage type 99 is associated with enhanced macrophage cytotoxicity. Infect Immun. 2003;71: 6068-6074. doi:10.1128/IAI.71.10.6068-6074.2003

15. Rabsch W, Andrews HL, Kingsley RA, Prager R, Tschäpe H, Adams LG, et al. Salmonella enterica serotype Typhimurium and its host-adapted variants. Infect Immun. 2002; 70: 2249-2255. doi:10.1128/IAI.70.5.2249-2255.2002

16. Woolhouse MEJ, Taylor LH, Haydon DT. Population biology of multihost pathogens. Science. 2001; 292: 1109-1112. doi:10.1126/science.1059026 
17. Okanga S, Cumming GS, Hockey PAR, Nupen L, Peters JL. Host specificity and cospeciation in avian haemosporidia in the Western Cape, South Africa. PLoS One. 2014; 9: e86382. doi:10.1371/journal.pone.0086382

18. Knowles SCL, Palinauskas V, Sheldon BC. Chronic malaria infections increase family inequalities and reduce parental fitness: Experimental evidence from a wild bird population. J Evol Biol. 2010; 23: 557-569. doi:10.1111/j.1420-9101.2009.01920.x

19. Lachish S, Knowles SCL, Alves R, Wood MJ, Sheldon BC. Fitness effects of endemic malaria infections in a wild bird population: the importance of ecological structure. $\mathbf{J}$ Anim Ecol. 2011; 80: 1196-1206. doi:10.1111/j.1365-2656.2011.01836.x

20. Webster JP, Woolhouse MEJ. Cost of resistance: Relationship between reduced fertility and increased resistance in a snail schistosome host-parasite system. Proc $\mathrm{R}$ Soc B Biol Sci. 1999; 266: 391-396. doi:10.1098/rspb.1999.0650

21. Dvorakova-Hortova K, Sidlova A, Ded L, Hladovcova D, Vieweg M, Weidner W, et al. Toxoplasma gondii decreases the reproductive fitness in mice. PLoS One. 2014; 9. doi:10.1371/journal.pone.0096770

22. Pasmans F, Baert K, Martel A, Bousquet-Melou A, Lanckriet R, De Boever S, et al. Induction of the carrier state in pigeons infected with Salmonella enterica subspecies enterica serovar typhimurium PT99 by treatment with florfenicol: A matter of pharmacokinetics. Antimicrob Agents Chemother. 2008; 52: 954-961. doi:10.1128/AAC.00575-07

23. Gharaibeh S, Mahmoud K, Al-Natour M. Field evaluation of maternal antibody transfer to a group of pathogens in meat-type chickens. Poult Sci. 2008; 87: 15501555. doi:10.3382/ps.2008-00119

24. Hamal K, Burgess S, Pevzner I, Erf G. Maternal Antibody Transfer From Dams to Their Egg Yolks, Egg Whites, and Chicks in Meat Lines of Chickens. Poult Sci. 2006; 
25. Patterson R, Youngner JS, Weigle WO, Dixon FJ. Antibody production and transfer to egg yolk in chickens. J Immunol. 1962; 89: 272-8.

26. Yosipovich R, Aizenshtein E, Shadmona R, Krispel S, Shuster E, Pitcovskiab J.

Overcoming the susceptibility gap between maternal antibody disappearance and autoantibody production. Vaccine. 2015; 33: 472-478. doi:10.1016/j.vaccine.2014.10.043

27. Shrestha S, Bjørnstad ON, King AA. Evolution of acuteness in pathogen

metapopulations: Conflicts between "classical" and invasion-persistence trade-offs.

28. Xiao Y, Bowers RG, Clancy D, French NP. Understanding the dynamics of 159-175. doi:10.1016/j.jtbi.2004.09.015

29. Dean D, Suchland RJ, Stamm WE. Evidence for Long-Term Cervical Persistence of

Chlamydia trachomatis by ompl Genotyping. J Infect Dis. 2000;182: 909-916. doi: $10.1086 / 315778$

30. Carey AJ, Huston WM, Cunningham KA, Hafner LM, Timms P, Beagley KW. doi:10.1111/aji.12093

31. Boyen F, Pasmans F, Van Immerseel F, Morgan E, Adriaensen C, Hernalsteens JP, et al. Salmonella Typhimurium SPI-1 genes promote intestinal but not tonsillar colonization in pigs. Microbes Infect. 2006;8: 2899-2907. doi:10.1016/j.micinf.2006.09.008

32. Nakamura M, Nagamine N, Takahashi T, Suzuki S, Kijima M, Tamura Y, et al. 
33. Verbrugghe E, Boyen F, Van Parys A, Van Deun K, Croubels S, Thompson A, et al.

Stress induced Salmonella Typhimurium recrudescence in pigs coincides with cortisol induced increased intracellular proliferation in macrophages. Vet Res. 2011;42: 118. doi:10.1186/1297-9716-42-118

34. Verbrugghe E, Dhaenens M, Leyman B, Boyen F, Shearer N, Van Parys A, et al. Host Stress Drives Salmonella Recrudescence. Sci Rep. 2016;6. doi:10.1038/srep20849

35. Gregory NG, Robins JK. A body condition scoring system for layer hens. New Zeal J Agric Res. 1998;41: 555-559. doi:10.1080/00288233.1998.9513338

36. Møller AP, Christe P, Erritzøe J, Mavarez J, Moller AP, Erritzoe J. Condition, Disease and Immune Defence. Oikos. 1998;83: 301-306. doi:10.2307/3546841

37. Griffiths R, Double MC, Orr K, Dawson RJG. A DNA test to sex most birds. Mol

38. Leyman B, Boyen F, Van Parys A, Verbrugghe E, Haesebrouck F, Pasmans F.

Salmonella Typhimurium LPS mutations for use in vaccines allowing differentiation of infected and vaccinated pigs. Vaccine. 2011;29: 3679-3685. doi:10.1016/j.vaccine.2011.03.004

39. Owen RD. Artificial Insemination of Pigeons and Doves. Poult Sci. 1941;20: 428-431. doi:10.3382/ps.0200428

40. Sontakke SD, Umapathy G, Sivaram V, Kholkute SD, Shivaji S. Semen characteristics, cryopreservation, and successful artificial insemination in the Blue rock pigeon (Columba livia). Theriogenology. 2004;62: 139-153.

41. Verbrugghe E, Boyen F, Gaastra W, Bekhuis L, Leyman B, Van Parys A, et al. The 
2012; 155: 115-127. doi:10.1016/j.vetmic.2011.09.012

42. Dickens MJ, Nephew BC, Romero LM. Captive European starlings (Sturnus vulgaris) in breeding condition show an increased cardiovascular stress response to intruders. Physiol Biochem Zool. 2006;79: 937-943. doi:10.1086/506007

43. Nephew BC, Romero LM. Behavioral, physiological, and endocrine responses of starlings to acute increases in density. Horm Behav. 2003; 44: 222-232. doi:10.1016/j.yhbeh.2003.06.002

44. Kitaysky AS, Wingfield JC, Piatt JF. Dynamics of food availability, body condition and physiological stress response in breeding Black-legged Kittiwakes. Funct Ecol. 1999;13: 577-584. doi:10.1046/j.1365-2435.1999.00352.x

45. Baker SW, Tucci ER, Felt SA, Zehnder A, Lentink D, Vilches-Moure JG. A Bird'sEye View of Regulatory, Animal Care, and Training Considerations Regarding Avian Flight Research. Comp Med. 2019;69: 169-178. doi:10.30802/AALAS-CM-18000033

46. Dunn IC, Wilson PW, D’Eath RB, Boswell T. Hypothalamic Agouti-Related Peptide mRNA is Elevated During Natural and Stress-Induced Anorexia. J Neuroendocrinol. 2015;27: 681-691. doi:10.1111/jne.12295

47. De Baere S, Rosendahl Larsen T, Devreese M, De Backer P, De Neve L, Fairhurst G, et al. Use of LC-MS-MS as an alternative to currently available immunoassay methods to quantitate corticosterone in egg yolk and albumen. Anal Bioanal Chem. 2015;407: 4351-4362. doi:10.1007/s00216-014-8269-7

48. Morrison CM, Dial SM, Day WA, Joens LA. Investigations of Salmonella enterica serovar Newport infections of oysters by using immunohistochemistry and knockout mutagenesis. Appl Environ Microbiol. 2012;78: 2867-2873. doi:10.1128/AEM.0745611 
49. Van Parys A, Boyen F, Volf J, Verbrugghe E, Leyman B, Rychlik I, et al. Salmonella Typhimurium resides largely as an extracellular pathogen in porcine tonsils, independently of biofilm-associated genes $\operatorname{csg} \mathrm{A}, \operatorname{csg} \mathrm{D}$ and $\operatorname{adr} \mathrm{A}$. Vet Microbiol. 2010;144: 93-99. doi:10.1016/j.vetmic.2009.12.021

50. Devleesschauwer B, Torgerson P, Charlier J, Levecke B, Praet N, Dorny P, et al. R Package 'prevalence': Tools for prevalence assessment studies. 2014; R package version 0.4.0. http://cran.r-project.org/package=prevalence

51. Rouffaer LO, Strubbe D, Teyssier A, Salleh Hudin N, Van den Abeele A-M, Cox I, et al. Effects of urbanization on host-pathogen interactions, using Yersinia in house sparrows as a model. PLoS One. 2017;12: e0189509. doi:10.1371/journal.pone.0189509

52. Bates D, Mächler M, Bolker BM, Walker SC. Fitting linear mixed-effects models using lme4. J Stat Softw. 2015;67: 1-48. doi:10.18637/jss.v067.i01

53. Hyndman RJ, Khandakar Y. Automatic time series forecasting: The 'forecast' package for R. J Stat Softw. 2008;27: 1-22. doi:10.18637/jss.v027.i03

54. Hothorn T, Bretz F, Westfall P. Simultaneous inference in general parametric models. Biom J. 2008; 50: 346-363. doi:10.1002/bimj.200810425 


\section{Supporting Information}

842 S1-7 Tables. Detailed statistical results of all analyses performed.

843

844 S1 Fig. Immunohistochemical staining of the ovary (A) and testes (B) of pigeons after

845 experimental inoculation with $1 \times 10^{8}$ CFU Salmonella Typhimurium varitas Copenhagen

846 DAB 69.

847

848 S2 Fig. Temporal dynamics of serum anti-Salmonella antibodies in pigeons, experimentally infected with $10^{8}$ CFU Salmonella Typhimurium varitas Copenhagen DAB69.

850

851 S3 Fig. Serum anti-Salmonella antibodies present in chicks from a Salmonella negative and a

852 Salmonella positive group at day 0,14 and 28 of age during both breeding periods.

853

854 S4 Fig. Serum anti-Salmonella antibodies present in pigeons from a Salmonella negative and 855 a Salmonella positive flock $(\mathrm{n}=16)$, before and 14 days after experimental inoculation with $85610^{3} \mathrm{CFU}$ of Salmonella Typhimurium varitas Copenhagen DAB69. 